\title{
The effect of interdisciplinary and diversified health education combined with personalized nutrition intervention on FPG, 2hPG, SDS, SAS scores and the pregnancy outcomes of gestational diabetes mellitus
}

\author{
Guo-Hong Zhu, Yun Xu, Li Zou, Qing Zhou, Li-Juan Zhou \\ Taizhou People's Hospital, Taihu Road, Medical High-tech Zone, Taizhou, China
}

\begin{abstract}
Objectives: This study aimed to explore the effect of interdisciplinary and diversified health education combined with personalized nutrition intervention on FPG, 2hPG, SDS, SAS scores and pregnancy outcome of gestational diabetes mellitus (GDM).

Material and methods: A total of 180 GDM patients, who were admitted to our hospital between June 2019 and June 2020, were enrolled as the research subjects and randomly divided into two groups: a research group and a control group $(n=90$, each). The patients in the control group received routine care while the patients in the research group received interdisciplinary and diversified health education combined with personalized nutrition intervention. The fasting blood-glucose (FPG), two-hour postprandial blood glucose (2hPBG), glycated hemoglobin ( $\mathrm{HbA1C})$, SDS, SAS scores, and pregnancy outcome of the two groups of pregnant women were analyzed and compared.

Results: The differences in the levels of $\mathrm{FBG}, 2 \mathrm{hPBG}$ and $\mathrm{HbA} 1 \mathrm{C}$ between the two groups before nursing were not statistically significant. After nursing, the levels of $\mathrm{FBG}, 2 \mathrm{hPBG}$, and $\mathrm{HbA1C}$ of the two groups of patients decreased, and the differences in each group before and after intervention were statistically significant. These indexes were lower in the research group than in the control group, the differences being statistically significant. There were no significant differences between the two groups in SAS and SDS scores before nursing, but there were statistically significant differences after nursing. The incidence of unfavorable pregnancy outcome was lower in the research group (8.89\%) than in the control group (14.44\%), but the difference was not statistically significant $(p>0.05)$.
\end{abstract}

Conclusions: Interdisciplinary and diversified health education combined with personalized nutrition intervention can effectively reduce FPG, $2 \mathrm{hPG}$, SDS, and SAS scores in GDM women.

Key words: gestational diabetes mellitus; health education; nutrition intervention; SDS and SAS scores; pregnancy outcome

Ginekologia Polska

\section{INTRODUCTION}

Gestational diabetes mellitus (GDM) is a common disease during pregnancy. At present, diabetes mellitus screening is included in routine pregnancy examinations. The disease refers to when a pregnant woman has a normal blood glucose level at the outset of her pregnancy but develops an abnormal blood glucose level during the gestation period, most commonly in mid-late pregnancy [1, 2]. With the changes in people's dietary structure and lifestyle, a high fat, high carbohydrate diet has appeared, resulting in overeating and the occurrence of chronic diseases. In particular, the number of diabetics is rapidly increasing. The
American Diabetes Association (ADS) pointed out in the "2014 Diabetes Diagnostic Criteria" [3] that the incidence of GDM has increased from $5-6 \%$ to $15-20 \%$. Current reports reveal that the incidence of GDM in China has reached $18.9 \%$ [4], which is one of the highest in the world. With the opening of the two-child policy, and the consequent increase in the number of high-risk pregnant women, those who are older or obese, for example, the incidence of GDM in China has risen even higher. GDM is a serious threat to maternal and infant health and often results in unfavorable pregnancy outcomes, such as maternal infection, dystocia, postpartum hemorrhage, macrosomia, fetal distress, neona-

\section{Corresponding author:}

Li-Juan Zhou

Taizhou People's Hospital, NNo. 366, Taihu Road, Medical High-tech Zone, 225300 Taizhou, China e-mail:zlj_zhou09@163.com 
tal hypoglycemia and even long-term eye disease. It can also cause many long-term complications for mothers and their offspring. An epidemiological study revealed that the incidence of obesity in adulthood in the offspring of GDM women was twice that of normal pregnant women, and the incidence of metabolic syndrome was four times greater [5]. The harm GDM can cause to mother and child should not be ignored, and timely diagnosis and appropriate treatment are needed. In this study, the effect of interdisciplinary and diversified health education combined with personalized diet intervention on GDM was analyzed in order to lay the foundation for future clinical application.

\section{MATERIAL AND METHODS}

\section{General information}

A total of $180 \mathrm{GDM}$ patients, who were admitted to our hospital from June 2019 to June 2020, were enrolled as the research subjects, and randomly divided into two groups: a research group and a control group ( $n=90$, each). The research group ranged from 22 to 41 years of age, with an average age of $35.52 \pm 8.11$, and the gestational age was 24-29 weeks, with an average of $26.94 \pm 1.78$ weeks. The age of the women in the control group ranged from $21-$ 42 years, with an average age of $35.13 \pm 8.09$ years, and the gestational age was 25-29 weeks, with an average of $26.94 \pm 1.82$ weeks. There was no significant difference in age, gestational age and other general data between the two groups $(P>0.05)$, hence they were comparable.

\section{Inclusion and exclusion criteria}

Inclusion criteria: 1) Pregnant women who met the GDM diagnostic criteria in the Guidelines for Diagnosis and Treatment of Gestational Diabetes Mellitus [3] after the assessment of all items; 2 ) The pregnant woman and/or her family member voluntarily signed the informed consent for the trial.

Exclusion criteria: 1) Pregnant women who were diagnosed with diabetes mellitus before pregnancy; 2 ) pregnant women with abnormal indexes of blood glucose and $\mathrm{HbA} 1 \mathrm{C}$ for various other reasons; 3) pregnant women with other organic diseases, such as liver or kidney dysfunction; and 4) pregnant women with progressive confusion.

\section{Material and methods}

Control group: Pregnant women in the control group received routine care, including prenatal health education, unified diet, and monitoring of blood glucose and weight by the patients themselves. Psychological guidance and exercise guidance were given if necessary.

Research group: Pregnant women in the research group received interdisciplinary and diversified health education combined with personalized diet intervention [6]. A team of doctors, nutritionists, psychological counselors and reha- bilitation nurses was established, and an education plan was devised for each patient. With their agreement, one-to-one guidance or group teaching was available, and interdisciplinary work helped patients to fully understand the etiology and mechanism of GDM, and how self-management and self-control could benefit them. Personalized diet guidance was also given; a retrospective 24-hour dietary survey was conducted, the results fed into the Chinese Nutrition Analysis Software Package, the daily caloric and nutrient indexes evaluated, and recipes developed for each woman, who was then monitored.

\section{Observation indexes}

The following indexes of the women in the two groups were observed and recorded: 1) Fasting blood glucose (FPG) and 2-hour postprandial blood glucose (2hPBG); blood glucose was measured using the glucose oxidase method. After the blood was processed, serum was obtained and added to a prepared peroxidase and enzyme phenol mixture. This underwent a water bath at $37^{\circ} \mathrm{C}$ for 15 minutes and was then measured using a UV spectrophotometer (model: DR6000, manufacturer: American hash) at the water level reference of $505 \mathrm{~nm}$. Glucose was calculated according to a standard curve. 2) Measurement of HbAlc: An immunoagglutination assay was performed. A glycosylated hemoglobin test kit was operated in strict accordance with the instructions (manufacturer: Shanghai Jining biological Co., Ltd.). The serum was placed in the kit for incubation at $37^{\circ} \mathrm{C}$, then $\mathrm{HbAlc}$ was determined and calculated with the biochemical analyzer (model: Attune NxT, manufacturer:Thermo Fisher). 3) Negative psychological evaluation: self-rating anxiety scale (SAS) and self-rating depression scale (SDS): these tables employ a four-grade scoring method, with a total score of 100 points, the degree of anxiety and depression being directly proportional to the score. 4) Pregnancy outcome:The incidences of neonatal hypoglycemia, caesarean section, gestational hypertension, macrosomia, premature delivery, hydramnios and postpartum hemorrhage in the two groups were statistically analyzed. Incidence $=$ number of cases/total cases $\times 100 \%$.

\section{Statistical analysis}

Statistical software SPSS20.00 was used for analysis. Measurement data were expressed as mean \pm standard deviation $(x \pm S D)$ or median (25th percentile, 75th percentile), compared using two independent sample t-tests or non-parametrical test. Count data were expressed as a percentage (\%) and compared using a Chi-square test. $\mathrm{P}<0.05$ was considered statistically significant.

\section{RESULTS}

Control of blood glucose and $\mathrm{HbA} 1 \mathrm{C}$ before and after nursing in the two groups 
The differences in the levels of FBG, $2 \mathrm{hPBG}$ and $\mathrm{HbA} 1 \mathrm{C}$ before nursing between the two groups were not statistically significant $(P>0.05)$; after nursing, the levels of FBG, $2 \mathrm{hPBG}$ and $\mathrm{HbA} 1 \mathrm{C}$ in the two groups had decreased significantly. There were significant differences before and after the intervention, and the levels of these indexes were significantly lower in the research group than in the control group, the differences being statistically significant (Tab. 1).

\section{A comparison of SAS and SDS scores before and after nursing}

As shown in Table 2, before nursing, there were no significant differences between the two groups in SAS and SDS scores $(P>0.05)$. However, after nursing there were significant differences between the two groups in both scores $(P<0.05)$.

\section{A comparison of the incidence of unfavorable pregnancy}

As shown in Table 3, the incidence of unfavorable pregnancy was $8.89 \%$ in the research group and $14.44 \%$ in the control group. In other words, it was lower in the research group than in the control group, but the difference was not statistically significant $(P>0.05)$.

\section{DISCUSSION}

Clinical research into GDM is at a preliminary stage, since the pathogenesis is relatively complex, and more data are needed [7]. During fetal growth, nutrients are mainly absorbed through the placenta, and the hormone levels of pregnant women fluctuate to a certain extent throughout pregnancy. In the mid-late pregnancy, the insulin sensitivity of pregnant women noticeably decreases. Some recent studies have concluded that if the women do not self-regulate, there is a risk of increased blood glucose. Although corresponding drug control can alleviate the degree to some extent, effective health guidance and diet are fundamental to controlling the situation [8].

At present, the "five carriages" method is used as a means of treating diabetes mellitus: health education, blood glucose self-monitoring, medical nutrition intervention, exercise therapy and drug therapy. GDM high-risk pregnant women account for the main group of women with diabetes mellitus and metabolic syndrome in the short-term, and long-term, postpartum. The purpose of health education during pregnancy is not only to improve unfavorable pregnancy outcomes, but also to help pregnant women alter their unhealthy lifestyles, and to avoid or prevent the occurrence of long-term postpartum complications. The develop-

\begin{tabular}{|c|c|c|c|c|c|c|c|}
\hline \multirow{2}{*}{ Groups } & \multirow{2}{*}{$\mathbf{n}$} & \multicolumn{2}{|c|}{ FBG( $\mathrm{mmol} / \mathrm{L})$} & \multicolumn{2}{|c|}{ 2hPBG( $\mathrm{mmol} / \mathrm{L})$} & \multicolumn{2}{|l|}{ HbA1C (\%) } \\
\hline & & Before & after* & Before & after* & Before & after* \\
\hline research group & 90 & $11.66 \pm 1.49$ & $5.64 \pm 0.92 \#$ & $17.70 \pm 3.11$ & $6.70 \pm 0.97 \#$ & $8.79 \pm 1.19$ & $5.88 \pm 0.69 \#$ \\
\hline control group & 90 & $11.75 \pm 1.52$ & $6.88 \pm 1.12 \#$ & $17.87 \pm 3.13$ & $8.91 \pm 1.02 \#$ & $8.85 \pm 1.33$ & $6.75 \pm 0.73 \#$ \\
\hline
\end{tabular}

*The difference between the research group and the control group is significant $(p<0.05)$; \#The difference between the research group or the control group after the intervention and before the intervention is significant $(p<0.05)$

\begin{tabular}{|c|c|c|c|c|c|}
\hline \multirow{2}{*}{ groups } & \multirow{2}{*}{$\mathbf{n}$} & \multicolumn{2}{|l|}{ SAS score } & \multicolumn{2}{|l|}{ SDS score } \\
\hline & & Before & after* & Before & after* \\
\hline research group & 90 & $56.86(43.12,70.52)$ & $28.69(18.18,39.10)$ & $62.96(51.82,75.32)$ & $27.96(17.66,38.42)$ \\
\hline control group & 90 & $56.78(42.92,71.52)$ & $37.96(26.84,50.16)$ & $63.02(51.26,76.18)$ & $40.94(29.16,52.82)$ \\
\hline
\end{tabular}

*The difference between the research group and the control group is significant $(p<0.05)$

\begin{tabular}{|l|l|l|l|l|l|l|l|l|}
\hline \multicolumn{2}{|l}{ Table 3. A comparison of the incidence of unfavorable pregnancy between the two groups } \\
\hline groups & $\mathbf{n}$ & $\begin{array}{l}\text { Premature } \\
\text { delivery }\end{array}$ & Macrosomia & $\begin{array}{l}\text { Postpartum } \\
\text { bleeding }\end{array}$ & $\begin{array}{l}\text { Cesarean } \\
\text { section }\end{array}$ & Hydramnios & $\begin{array}{l}\text { Neonatal } \\
\text { hypoglycemia }\end{array}$ & $\begin{array}{l}\text { Total number of } \\
\text { incidents (rate) }\end{array}$ \\
\hline research group & 90 & 1 & 1 & 1 & 2 & 1 & 2 & $8(8.89 \%)$ \\
\hline control group & 90 & 2 & 2 & 1 & 3 & 2 & 3 & $13(14.44 \%)$ \\
\hline
\end{tabular}

*The difference between the research group and the control group is significant $(p<0.05)$. 
ment of health education for patients with GDM has been proven to be an important diabetes treatment measure besides drug treatment [9]. Medical nutrition intervention (MNT), which is the basis of diabetes treatment, is an essential measure to prevent and control diabetes at any stage, and a basic management method throughout the whole process of the disease. In 1994, the American Dental Association (ADA) put forward the term"medical nutritional therapy (MNT)" [10] and emphasized the importance of personalized intervention in 2006. A previous study revealed that strict management of mild hyperglycemia during pregnancy could significantly improve maternal and infant outcomes (A-level evidence) [11]. Intensive GDM patients can prevent the occurrence of complications during the perinatal period by changing their unfavorable diet and lifestyle. This economical measure is worthy of promotion by clinicians.

Interdisciplinary and diversified health education, combined with the personalized diet intervention model, effectively considers all aspects of the issues that face GDM pregnant women during their pregnancy. The interdisciplinary and diversified health education team include key personnel who have mastered the knowledge of different disciplines so they can understand the specific situation of each individual patient and teach them accordingly. They can enable patients to control their own condition by working and resting as appropriate and taking the proper exercise [12-14]. And, when patients are anxious, guidance can be given to ensure their psychological well-being is also being addressed [10]. Personalized dietary guidance for clinical treatment should be given according to the actual situation of each pregnant women and cannot be generalized. For example, the actual blood glucose, body weight and nutritional status of the pregnant women were analyzed as well as their daily lives and eating habits to provide personalized and scientific diet guidance. This included suggestions concerning daily calorie intake, and a reasonable adjustment of food ingredients, to control their weight increase. These programs had a positive impact on the effective prevention of unfavorable outcomes.

In this study, there were no significant differences in FBG, 2hPBG, HbA1C, SAS and SDS scores between the two groups before nursing. However, after nursing all the indexes had fallen, they were significantly lower in the research group than in the control group. The final unfavorable pregnancy outcomes were analyzed and were also found to be lower in the research group than in the control group. The results suggest that the implementation of interdisciplinary diversified health education, combined with personalized diet intervention, cannot only effectively reduce blood glucose and other indicators of diabetes, but they also actively affect the psychological state of patients and reduce unfavorable pregnancy outcomes. Therefore, they should be used widely in the clinical treatment of GDM patients.

\section{Funding}

Six Talent Peak Project (WSN-336)

Taizhou Science and Technology Support Plan (Social Development) Project (TS201902)

\section{Conflict of interests}

The Authors declare that they have no conflict of interests.

\section{REFERENCES}

1. Kusinski LC, Murphy HR, Rolfe ED, et al. Erratum: dietary intervention in pregnant women with gestational diabetes; protocol for the DiGest randomised controlled trial; . Nutrients. 2020; 12(6), doi: 10.3390/nu12061793, indexed in Pubmed: 32560576.

2. Martis R, Crowther CA, Shepherd E, et al. Treatments for women with gestational diabetes mellitus: an overview of Cochrane systematic reviews. Cochrane Database Syst Rev. 2018; 8: CD012327, doi: 10.1002/14651858.CD012327.pub2, indexed in Pubmed: 30103263.

3. American Diabetes Association. Diagnosis and classification of diabetes mellitus[J]. Diabetes Care. 2014; 37(Suppl 1): s14-s80, doi: 10.2337/dc14-S081.

4. Wei Y, Yang H, Zhu W, et al. International Association of Diabetes and Pregnancy Study Group criteria is suitable for gestational diabetes mellitus diagnosis: further evidence from China. Chin Med J (Engl). 2014; 127(20): 3553-3556.

5. Clausen TD, Mathiesen ER, Hansen T, et al. Overweight and the metabolic syndrome in adult offspring of women with diet-treated gestational diabetes mellitus or type 1 diabetes. J Clin Endocrinol Metab. 2009; 94(7): 2464-2470, doi: 10.1210/jc.2009-0305, indexed in Pubmed: 19417040.

6. Nengzhen B. Exploring the impact of nutritional interventions on weight and pregnancy outcomes in patients with gestational diabetes mellitus[J]. Journal of Electrocardiogram (Electronic Edition). 2020; 9(3): 243-244.

7. Yaping S, Yuling T. Effect of individualized dietary interventions on blood glucose in gestational diabetes mellitus. Hebei Medical Journal. 2020; 32(12): 1653-1654.

8. Jinxin $L$, Sihong $L$. The importance analysis of individualized diet guidance on gestational diabetes mellitus. Clinical Journal of Chinese Medicine, 2018; 10(15): 75-76, doi: 10.3969/j.issn.1674-7860.2018.15.035.

9. Xinke $H$, Dongxi $H$, Nan F. Evaluation of effect assessment on case-management of women with gestational diabetes mellitus. American Journal of Nursing Science. 2019; 8(6): 313-316, doi: 10.11648/j. ajns.20190806.14.

10. American Diabetes Association. Standards of Medical Care in Diabetes--2010. Diabetes Care. 2009; 33(Supplement_1): S11-S61, doi: 10.2337/dc10-s011.

11. Landon MB, Spong CY, Thom E, et al. A Multicenter, randomized trial of treatment for mild gestational diabetes. Obstetric Anesthesia Digest. 2010; 30(4): 232, doi: 10.1097/01.aoa.0000389610.71133.ee.

12. Jiehui C. A study of the impact of individualized dietary management on pregnancy outcomes in patients with gestational diabetes mellitus. Journal of Youjiang Medical University for Nationalities. 2019; 36(4): 639-641.

13. Tian R. Effect of health education and dietary care on blood glucose levels in pregnant women with gestational diabetes mellitus. Electronic Journal of Practical Clinical Nursing Science. 2020; 5(13).

14. Xiaojia Ma, Nanping X. Application value of health education in outpatient of gestational diabetes patients. Chinese and Foreign Medical Research. 2019; 17(35): 176-178. 